\title{
Long-distance travel for birthing among Indigenous and non-Indigenous pregnant people in Canada
}

\author{
Janet Smylie MD MPH, Kristen O’Brien MSc, Emily Beaudoin MD MPH, Nihaya Daoud MPH PhD, \\ Cheryllee Bourgeois BHSc Midwifery, Evelyn Harney George BA BSc Midwifery, \\ Kerry Bebee BA BHSc Midwifery, Chaneesa Ryan MA
}

Cite as: CMAJ 2021 June 21;193:E948-55. doi: 10.1503/cmaj.201903

CMAJ Podcasts: author interview at www.cmaj.ca/lookup/doi/10.1503/cmaj.201903/tab-related-content

\begin{abstract}
BACKGROUND: For Indigenous Peoples in Canada, birthing on or near traditional territories in the presence of family and community is of foundational cultural and social importance. We aimed to evaluate the association between Indigenous identity and distance travelled for birth in Canada.
\end{abstract}

METHODS: We obtained data from the Maternity Experiences Survey, a national population-based sample of new Canadian people aged 15 years or older who gave birth (defined as mothers) and were interviewed in 2006-2007. We compared Indigenous with non-Indigenous Canadian-born mothers and adjusted for geo- graphic and sociodemographic factors and medical complications of pregnancy using multivariable logistic regression. We categorized the primary outcome, distance travelled for birth, as 0 to 49,50 to 199 or $200 \mathrm{~km}$ or more.

RESULTS: We included 3100 mothers living in rural or small urban areas, weighted to represent 31100 (1800 Indigenous and 29300 non-Indigenous Canadian-born mothers). We found that travelling $200 \mathrm{~km}$ or more for birth was more common among Indigenous compared with nonIndigenous mothers (9.8\% v. $2.0 \%$, odds ratio [OR] 5.45, 95\% confidence interval [Cl] 3.52-8.48). In adjusted analyses, the associ- ation between Indigenous identity and travelling more than $200 \mathrm{~km}$ for birth was even stronger (adjusted OR 16.44, 95\% Cl 8.07-33.50) in rural regions; however, this was not observed in small urban regions (adjusted OR 1.04, 95\% Cl 0.37-2.91).

INTERPRETATION: Indigenous people in Canada experience striking inequities in access to birth close to home compared with non-Indigenous people, primarily in rural areas and independently of medical complications of pregnancy. This suggests inequities are rooted in the geographic distribution of and proximal access to birthing facilities and providers for Indigenous people.
A c cess to birth close to home, surrounded by loved ones, is taken for granted by most Canadians. The societal importance of family support during birthing has been highlighted during the severe acute respiratory syndrome and COVID-19 pandemics, despite the known and potentially fatal risks to hospital visitors, because people in labour have been one of the few patient groups exempt from visitor restrictions. ${ }^{1,2}$ For residents living in rural areas of Canada, long-distance travel for birth is a reality that is becoming increasingly common in some regions because of closures of obstetrical services in smaller community hospitals. ${ }^{3}$ This is only partially mitigated by the revitalization of rural midwifery practice..$^{4,5}$

Emerging evidence shows that the frequency of adverse medical events during labour and delivery for rural populations is similar for births that take place close to home and births for which people travel because of an absence of services close to home. ${ }^{3,4,6}$ Less is known about the impacts of travel for birth on breastfeeding rates, maternal mental health and family functioning. Several studies have documented the negative impacts of birthing away from home with respect to maternal satisfaction and birth experience. ${ }^{7-10}$ This evidence is particularly compelling for Indigenous populations for whom birthing on or near traditional territories in the presence of family and community is a long-standing practice of foundational cultural and social importance that contributes to well-being, cultural continuity and kinship. ${ }^{7-12}$

The striking isolation, family disruption and racism experienced by Indigenous people who are forced to travel alone for 
birth as a result of externally imposed federal "evacuation for birth" policies ${ }^{11}$ has been met with a series of policy initiatives to support return of birth to rural and remote Indigenous communities. ${ }^{13-15}$ In April 2017, then federal Minister of Health Dr. Jane Philpott, committed to "a path to be able to return the cries of birth" to Indigenous communities and funding to support travel for a companion when Indigenous people living in rural and remote areas needed to travel away from home for birth. ${ }^{16}$ Before 2017, Indigenous pregnant people often travelled and birthed away from home alone without family or community support, because escorts were not deemed medically necessary. Although these initiatives have improved access to Indigenous perinatal programming and Indigenous birth attendant support in some local areas, over the past decade there has not been any substantial expansion of Indigenous birthing facilities outside of urban centres in Canada and at least 1 remote Indigenous birthing facility has closed. ${ }^{17}$

Given this dynamic policy context, the national scope and Indigenous identifiers in the Canadian Maternity Experiences Survey (MES) provides a unique opportunity to quantify how often Indigenous and non-Indigenous people are travelling away from home for birth and to evaluate the association between Indigenous and non-Indigenous identity and distance travelled for birth in Canada.

\section{Methods}

\section{Study design and cohort}

We analyzed data from the 2006-2007 MES, ${ }^{18,19}$ Canada's most recent national survey of maternity health, which referred to participants as mothers and women. The MES recruited mothers aged 15 years or older who had a singleton live birth in Canada 5-9 months before the survey date. The MES sample was randomly selected from the Canadian Census and designed to be a representative stratified random sample. The survey was administered by trained interviewers using a computer-assisted telephone interview application, and interviews were conducted in English, French and 13 additional languages. First Nations mothers living on reserve, and all mothers who were institutionalized or whose children were not living with them due to child protection agency involvement were not included in this survey. Additional information regarding the methods and results of the MES have been described by others. ${ }^{18,19}$

We included Indigenous and non-Indigenous Canadian-born mothers who had complete information for travel distance and other sociodemographic factors and lived in rural or small urban areas in the cohort for our main adjusted analysis. We chose non-Indigenous Canadian-born mothers as our comparison cohort because immigrant Canadians are known to have disparities in access to health care and perinatal outcomes compared with Canadian-born mothers. ${ }^{20,21}$ Owing to the small numbers of mothers who travelled more than $50 \mathrm{~km}$ for birth in urban areas, we excluded those living in urban areas from our analysis. Disaggregation by First Nations, Inuit and Métis identity was not possible for this analysis because of the small sample size.

\section{Primary outcome}

Our primary outcome was the distance that the mother had to travel to give birth. The MES asked whether or not they travelled to give birth to their baby and to select the distance travelled to another city, town or community for the birth. The distance travelled was then categorized as $0-49,50-199$, or $200 \mathrm{~km}$ or more. We chose our first cut point to represent more than 30 minutes of travel by road to distinguish between local and nonlocal travel, which has clinical implications in the event of urgencies or emergencies linked to labour and delivery. Our second cut point was chosen to represent more than 2 hours of travel by road, which we identified as a threshold over which the feasibility of family and community birthing supports commuting back and forth becomes limited. The middle category, 50-199 km, could potentially be a feasible commute for some, based on patterns of commuting for work in large urban centres.

\section{Independent variables}

Our main independent variable of interest was whether or not the mother identified as Indigenous. Identity was determined by asking all mothers who had responded yes to the question "Were you born in Canada?" the following: "Are you an Aboriginal person, that is, First Nations, Métis or Inuit?" If the response was no, then they were classified as non-Indigenous, Canadian born; they were our comparison group for reasons described previously.

The MES used Statistics Canada definitions for categories of "area size of residence" to classify maternal residency as rural, urban (population < 100000 , described hereafter as "small urban"] or urban (population $\geq 100000$, described hereafter as "large urban"), through the question: "What is your postal code?"

Drawing on the literature, we identified additional sociodemographic characteristics and variables of health care use that are known to influence labour and birth outcomes independently or that could contribute to travel for birth. 22,23 We evaluated and controlled for independent variables from these domains in our analysis. Sociodemographic variables included maternal age at birth (dichotomized as aged 15-24 and 25-50 yr, in keeping with categorizations commonly used in perinatal epidemiology and in previous analyses of MES data, as well as because occurrence of adverse birth outcomes is skewed toward the youngest and oldest); marital status (lone or cohabitating); level of education (less than high school, completed high school diploma or vocational certificate, or completed more than high school or vocational certificate); before-tax income (at or below the low income cut-off, above the low income cut-off or missing); and parity (1 child, 2 children or more). We chose low income cut-off over household income as the independent variable for socioeconomic position to better account for families with children. Factors regarding use of health care by the mother and child included: prelabour maternal hospital admissions during pregnancy (yes, no); delivery type (vaginal, including instrumental, noninstrumental and unknown instrumental; or cesarean delivery); other birth complications (yes, no); and infant admission 
to the neonatal intensive care unit (NICU) right after birth (yes, no). We also considered several variables for maternal experience and relations that we theorized could be associated with travel for birth (Table 1 ) but did not include them in our adjusted models because they were considered to be upstream in the causal pathway or occurring after travel for birth.

\section{Statistical analysis}

Our preliminary analysis included Indigenous and non-Indigenous Canadian-born mothers from large urban, small urban and rural areas. To begin, we constructed weighted descriptive statistics for all variables. We evaluated the association between Indigenous or non-Indigenous identity and each of the independent variables using $\chi^{2}$ tests and univariate multinomial logistic regression models.

Based on the known geographic distributions of Indigenous and non-Indigenous populations in Canada, ${ }^{24,25}$ we determined that urban versus rural residency may be an effect modifier to Indigenous and non-Indigenous identity and travel distance. To evaluate effect modification by Indigenous identity and travel distance, we conducted multivariate logistic regression on models stratified by urban and rural residency. Support for the stratification approach was shown by the presence of significant interaction terms in bivariate analyses between geographic region and Indigenous/non-Indigenous identity. In addition, the number of mothers who travelled $50 \mathrm{~km}$ or more to give birth and who lived in large urban areas was small. Because of small cell counts and lack of variance, we focused our adjusted models on the relation between Indigenous identity and travel distance for mothers from rural and small urban areas only. To determine factors associated with travel distance, we constructed multivariate multinomial logistic regression models within each stratum, with travel distance as the outcome. The first model evaluated the association

Table 1 (part 1 of 2): Characteristics of Indigenous and non-Indigenous Canadian-born mothers in small urban and rural areas and the total cohort

\begin{tabular}{|c|c|c|c|c|c|c|c|c|c|}
\hline \multirow[b]{2}{*}{ Characteristic } & \multirow[b]{2}{*}{$\begin{array}{l}\text { No. }(\%) \text { of } \\
\text { Indigenous } \\
\text { mothers } \\
n=600\end{array}$} & \multirow{2}{*}{$\begin{array}{c}\begin{array}{c}\text { Rural area } \\
n=12800^{\star}\end{array} \\
\text { No. (\%) of } \\
\text { non-Indigenous } \\
\text { mothers } \\
n=12200\end{array}$} & \multirow[b]{2}{*}{$p$ value } & \multicolumn{3}{|c|}{$\begin{array}{l}\text { Small urban area } \\
\qquad n=18300^{\star}\end{array}$} & \multicolumn{3}{|c|}{$\begin{array}{l}\text { Total cohort } \\
n=31100^{\star} \dagger\end{array}$} \\
\hline & & & & $\begin{array}{l}\text { No. }(\%) \text { of } \\
\text { Indigenous } \\
\text { mothers } \\
n=1200\end{array}$ & $\begin{array}{c}\text { No. }(\%) \text { of } \\
\text { non-Indigenous } \\
\text { mothers } \\
n=17100\end{array}$ & $p$ value & $\begin{array}{l}\text { No. }(\%) \text { of } \\
\text { Indigenous } \\
\text { mothers } \\
n=1800\end{array}$ & $\begin{array}{c}\text { No. }(\%) \text { of } \\
\text { non-Indigenous } \\
\text { mothers } \\
n=29300\end{array}$ & $p$ value \\
\hline \multicolumn{10}{|l|}{ Age of mother at birth, yr } \\
\hline $15-24$ & $230(38.4)$ & $1964(16.1)$ & $<0.001$ & $444(37.0)$ & $3164(18.5)$ & $<0.001$ & $675(37.5)$ & $5128(17.5)$ & $<0.001$ \\
\hline $25-50$ & $370(61.6)$ & $10236(83.9)$ & & $756(63.0)$ & $13937(81.5)$ & & $1125(62.5)$ & $24173(82.5)$ & \\
\hline \multicolumn{10}{|l|}{ Marital status } \\
\hline Lone & $96(16.0)$ & $781(6.4)$ & 0.003 & $352(29.3)$ & $1402(8.2)$ & $<0.001$ & $446(24.8)$ & $2198(7.5)$ & $<0.001$ \\
\hline Cohabitating & $504(84.0)$ & $11419(93.6)$ & & $848(70.7)$ & $15698(91.8)$ & & $1354(75.2)$ & $27103(92.5)$ & \\
\hline \multicolumn{10}{|l|}{ Education } \\
\hline Less than high school & $136(22.6)$ & $939(7.7)$ & $<0.001$ & $252(21.0)$ & $1471(8.6)$ & $<0.001$ & $387(21.5)$ & $2403(8.2)$ & $<0.001$ \\
\hline $\begin{array}{l}\text { High school diploma or } \\
\text { vocational certificate }\end{array}$ & $270(45.0)$ & $3745(30.7)$ & & $566(47.2)$ & $4925(28.8)$ & & $837(46.5)$ & $8673(29.6)$ & \\
\hline $\begin{array}{l}\text { Greater than high school diploma } \\
\text { or vocational certificate }\end{array}$ & $194(32.4)$ & $7515(61.6)$ & & $382(31.8)$ & $10705(62.6)$ & & $576(32.0)$ & $18225(62.2)$ & \\
\hline \multicolumn{10}{|l|}{ Household income, \$ } \\
\hline 0-29999 & $192(32.0)$ & $1696(13.9)$ & $<0.001$ & $492(41.0)$ & $2172(12.7)$ & $<0.001$ & $682(37.9)$ & $3868(13.2)$ & $<0.001$ \\
\hline $30000-59999$ & $221(36.8)$ & 3965 (32.5) & & $241(20.1)$ & $5147(30.1)$ & & $464(25.8)$ & $9112(31.1)$ & \\
\hline$\geq 60000$ & $152(25.4)$ & $5990(49.1)$ & & $340(28.3)$ & $9029(52.8)$ & & $491(27.3)$ & $15031(51.3)$ & \\
\hline Missing & $35(5.8)$ & $549(4.5)$ & & $127(10.6)$ & $752(4.4)$ & & $162(9.0)$ & $1319(4.5)$ & \\
\hline \multicolumn{10}{|l|}{ Before-tax income } \\
\hline At or below the low-income cut-off & $184(30.7)$ & $1415(11.6)$ & $<0.001$ & $464(38.7)$ & $2189(12.8)$ & $<0.001$ & $648(36.0)$ & $3604(12.3)$ & $<0.001$ \\
\hline Above the low-income cut-off & $381(63.5)$ & $10236(83.9)$ & & $608(50.7)$ & $14142(82.7)$ & & $990(55.0)$ & $24378(83.2)$ & \\
\hline Missing & $35(5.8)$ & $549(4.5)$ & & $127(10.6)$ & $770(4.5)$ & & $162(9.0)$ & $1319(4.5)$ & \\
\hline \multicolumn{10}{|l|}{ Parity } \\
\hline 1 child & $257(42.9)$ & 4819 (39.5) & 0.6 & $430(35.8)$ & $7285(42.6)$ & 0.2 & $688(38.2)$ & $12101(41.3)$ & 0.4 \\
\hline 2 children or more & $343(57.1)$ & $7381(60.5)$ & & $770(64.2)$ & $9815(57.4)$ & & $1112(61.8)$ & $17199(58.7)$ & \\
\hline \multicolumn{10}{|l|}{ Social support during pregnancy } \\
\hline No support or some & $318(53.0)$ & $4624(37.9)$ & 0.02 & $444(37.0)$ & $6789(39.7)$ & 0.6 & 765 (42.5) & $11427(39.0)$ & 0.4 \\
\hline All times & $282(47.0)$ & $7576(62.1)$ & & $756(63.0)$ & $10311(60.3)$ & & $1035(57.5)$ & $17873(61.0)$ & \\
\hline \multicolumn{10}{|l|}{ Stressful events in past $12 \mathrm{mo}$} \\
\hline Not stressful & $269(44.9)$ & $5514(45.2)$ & 0.7 & $391(32.6)$ & $7404(43.3)$ & 0.01 & $662(36.8)$ & $12921(44.1)$ & 0.01 \\
\hline Somewhat stressful & $246(41.0)$ & $5368(44.0)$ & & $565(47.1)$ & $7883(46.1)$ & & $810(45.0)$ & $13244(45.2)$ & \\
\hline Very stressful & $85(14.1)$ & $1318(10.8)$ & & $244(20.3)$ & $1813(10.6)$ & & $328(18.2)$ & $3135(10.7)$ & \\
\hline
\end{tabular}




\begin{tabular}{|c|c|c|c|c|c|c|c|c|c|}
\hline \multirow[b]{2}{*}{ Characteristic } & \multicolumn{3}{|c|}{$\begin{array}{l}\text { Rural area } \\
n=12800^{\star}\end{array}$} & \multicolumn{3}{|c|}{$\begin{array}{l}\text { Small urban area } \\
\qquad n=18300^{\star}\end{array}$} & \multicolumn{3}{|c|}{$\begin{array}{l}\text { Total cohort } \\
n=31100^{\star} \dagger\end{array}$} \\
\hline & $\begin{array}{l}\text { No. }(\%) \text { of } \\
\text { Indigenous } \\
\text { mothers } \\
n=600\end{array}$ & $\begin{array}{c}\text { No. }(\%) \text { of } \\
\text { non-Indigenous } \\
\text { mothers } \\
n=12200\end{array}$ & $p$ value & $\begin{array}{l}\text { No. }(\%) \text { of } \\
\text { Indigenous } \\
\text { mothers } \\
n=1200\end{array}$ & $\begin{array}{c}\text { No. }(\%) \text { of } \\
\text { non-Indigenous } \\
\text { mothers } \\
n=17100\end{array}$ & $p$ value & $\begin{array}{l}\text { No. }(\%) \text { of } \\
\text { Indigenous } \\
\text { mothers } \\
n=1800\end{array}$ & $\begin{array}{c}\text { No. }(\%) \text { of } \\
\text { non-Indigenous } \\
\text { mothers } \\
n=29300\end{array}$ & $p$ value \\
\hline \multicolumn{10}{|l|}{ Any abuse } \\
\hline No & $421(70.1)$ & $11126(91.2)$ & $<0.001$ & 859 (71.6) & $15082(88.2)$ & $<0.001$ & $1280(71.1)$ & $26194(89.4)$ & $<0.001$ \\
\hline Yes & $179(29.9)$ & $1074(8.8)$ & & $341(28.4)$ & $2018(11.8)$ & & $520(28.9)$ & $3106(10.6)$ & \\
\hline \multicolumn{10}{|c|}{ Travel distance to the hospital for birth, km } \\
\hline No travel and $<50$ & $314(52.4)$ & $9577(78.5)$ & $<0.001$ & $1049(87.4)$ & $15202(88.9)$ & 0.8 & $1357(75.4)$ & $24788(84.6)$ & $<0.001$ \\
\hline $50-200$ & $146(24.4)$ & $2367(19.4)$ & & $118(9.8)$ & $1556(9.1)$ & & $266(14.8)$ & $3926(13.4)$ & \\
\hline$\geq 200$ & $139(23.2)$ & $256(2.1)$ & & $34(2.8)$ & 325 (1.9) & & $176(9.8)$ & $586(2.0)$ & \\
\hline \multicolumn{10}{|l|}{ Birth satisfaction } \\
\hline Low & $53(8.8)$ & $927(7.6)$ & 0.9 & $90(7.5)$ & $1539(9.0)$ & 0.6 & $142(7.9)$ & $2461(8.4)$ & 0.8 \\
\hline Moderate & $64(10.7)$ & $1305(10.7)$ & & $104(8.7)$ & $1932(11.3)$ & & $169(9.4)$ & $3223(11.0)$ & \\
\hline High & $483(80.5)$ & $9967(81.7)$ & & $1006(83.8)$ & $13629(79.7)$ & & $1489(82.7)$ & $23587(80.5)$ & \\
\hline \multicolumn{10}{|l|}{ Satisfied with travel } \\
\hline Positive & 209 (34.9) & $2855(23.4)$ & 0.02 & $134(11.2)$ & $3215(18.8)$ & 0.06 & $347(19.3)$ & 6065 (20.7) & 0.08 \\
\hline Neutral & $81(13.5)$ & $2294(18.8)$ & & $73(6.1)$ & $1932(11.3)$ & & $155(8.6)$ & $4219(14.4)$ & \\
\hline Negative & $83(13.8)$ & $830(6.8)$ & & $53(4.4)$ & $616(3.6)$ & & $137(7.6)$ & $1465(5.0)$ & \\
\hline Missing/not applicable & $227(37.8)$ & $6210(50.9)$ & & $940(78.3)$ & $11337(66.3)$ & & $1161(64.5)$ & $17551(59.9)$ & \\
\hline \multicolumn{10}{|c|}{ Stay in hospital during pregnancy but before labour } \\
\hline Yes & $190(31.6)$ & $1574(12.9)$ & $<0.001$ & $318(26.5)$ & $2377(13.9)$ & 0.0005 & $508(28.2)$ & $3956(13.5)$ & $<0.001$ \\
\hline No & $410(68.4)$ & $10626(87.1)$ & & $882(73.5)$ & $14723(86.1)$ & & $1292(71.8)$ & $25345(86.5)$ & \\
\hline \multicolumn{10}{|l|}{ Delivery type } \\
\hline $\begin{array}{l}\text { Vaginal (including spontaneous } \\
\text { and instrumental) }\end{array}$ & $445(74.1)$ & $9089(74.5)$ & 0.9 & $833(69.4)$ & $12722(74.4)$ & 0.3000 & $1278(71.0)$ & $21799(74.4)$ & 0.3 \\
\hline Cesarean & $155(25.9)$ & $3111(25.5)$ & & $367(30.6)$ & $4378(25.6)$ & & $522(29.0)$ & $7501(25.6)$ & \\
\hline \multicolumn{10}{|l|}{ Other birth complications } \\
\hline Yes & $41(6.9)$ & $1061(8.7)$ & 0.6 & $76(6.3)$ & $1556(9.1)$ & 0.3000 & $117(6.5)$ & $2608(8.9)$ & \\
\hline No & $559(93.1)$ & $11139(91.3)$ & & $1124(93.7)$ & $15544(90.9)$ & & $1683(93.5)$ & $26692(91.1)$ & 0.2 \\
\hline \multicolumn{10}{|c|}{ Neonatal intensive care unit admission } \\
\hline Yes & $38(6.4)$ & $1342(11.0)$ & 0.2 & $128(10.7)$ & $2103(12.3)$ & 0.6 & $166(9.2)$ & $3457(11.8)$ & \\
\hline No & $562(93.6)$ & $10858(89.0)$ & & $1072(89.3)$ & $14997(87.7)$ & & $1634(90.8)$ & $25843(88.2)$ & 0.3 \\
\hline
\end{tabular}

between identity and travel distance. Each successive model evaluated the association of identity with travel distance while adjusting for a different sociodemographic, maternal health or infant health variable. Our final adjusted model included all factors, regardless of their statistical significance. All analyses were conducted using SAS 9.4. We applied bootstrapped weights to adjust for the complex study design and rounded stratum totals to the nearest 100th to protect the identity of participants.

\section{Ethics approval}

The MES was approved by Statistics Canada's Policy Committee. Our study was approved by the St. Michael's Hospital Research Ethics Board. Observing the standards for the secondary data analysis involving Indigenous Peoples, ${ }^{26}$ we conducted this analysis in partnership with the Native Women's Association of Canada and the National Aboriginal Council of Midwives.

\section{Results}

The MES obtained adequately completed responses from 6241 mothers (77.9\% of eligible sample), which was weighted to represent $76178 .{ }^{18} \mathrm{After}$ rounding values to ensure anonymity, 3100 mothers living in rural or small urban areas met our criteria, weighted to represent 31100 (1800 Indigenous and 29300 non-Indigenous Canadian-born mothers). Among Indigenous mothers, $34.2 \%$ lived in rural areas and $65.8 \%$ lived in small urban areas; among non-Indigenous mothers, $41.6 \%$ lived in rural areas and $58.4 \%$ lived in small urban areas $(p=$ 0.06). Descriptive analysis (Table 1) showed differences in demographic characteristics between Indigenous and nonIndigenous mothers across geographies of residence: small urban, rural, and small urban and rural combined. Across strata, Indigenous mothers were more likely to be younger, report a lone relationship status, have an income of less than 
Table 2: Unadjusted and adjusted multivariable odds of travelling $\mathbf{5 0 ~ k m}$ or more for birth among Indigenous and non-Indigenous Canadian-born mothers in rural residency

\begin{tabular}{|c|c|c|c|c|}
\hline \multirow[b]{3}{*}{ Variable } & \multicolumn{4}{|c|}{$\begin{array}{l}\text { Rural residence } \\
\qquad n=12805\end{array}$} \\
\hline & \multicolumn{2}{|c|}{$\begin{array}{c}\text { Travel } 50-199 \mathrm{~km} \text { v. no travel } \\
\text { or }<50 \mathrm{~km}\end{array}$} & \multicolumn{2}{|c|}{$\begin{array}{c}\text { Travel } \geq 200 \mathrm{~km} \mathrm{v.} \mathrm{no} \mathrm{travel} \\
\text { or }<50 \mathrm{~km}\end{array}$} \\
\hline & $\begin{array}{l}\text { Unadjusted }{ }^{\star} \text { OR } \\
(95 \% \mathrm{Cl})\end{array}$ & $\begin{array}{l}\text { Adjusted OR } \\
(95 \% \mathrm{Cl})\end{array}$ & $\begin{array}{l}\text { Unadjusted }{ }^{\star} \text { OR } \\
(95 \% \mathrm{Cl})\end{array}$ & $\begin{array}{l}\text { Adjusted OR } \\
\quad(95 \% \mathrm{Cl})\end{array}$ \\
\hline \multicolumn{5}{|l|}{ Ethnic identity } \\
\hline Indigenous v. non-Indigenous & $1.89(1.01-3.53)$ & $1.63(0.83-3.22)$ & $16.59(8.98-30.65)$ & $16.44(8.07-33.10)$ \\
\hline \multicolumn{5}{|l|}{ Age, yr } \\
\hline $15-24$ v. $25-50$ & $0.83(0.57-1.19)$ & $0.95(0.64-1.42)$ & $0.69(0.35-1.37)$ & $1.07(0.46-2.51)$ \\
\hline \multicolumn{5}{|l|}{ Marital status } \\
\hline Lone v. cohabitating & $1.54(0.91-2.61)$ & $1.15(0.63-2.08)$ & $1.62(0.74-3.58)$ & $0.82(0.30-2.23)$ \\
\hline \multicolumn{5}{|l|}{ Education } \\
\hline Less than high school & $1.52(0.91-2.54)$ & $1.38(0.79-2.41)$ & $1.73(0.79-3.80)$ & $1.03(0.45-2.36)$ \\
\hline High school diploma & $1.44(1.04-1.98)$ & $1.39(0.99-1.97)$ & $1.12(0.52-2.40)$ & $0.95(0.43-2.09)$ \\
\hline Postsecondary diploma or university & Ref. & & & \\
\hline \multicolumn{5}{|l|}{ Before-tax income } \\
\hline At or below low-income cut-off & $1.34(0.90-2.01)$ & $1.16(0.72-1.87)$ & $1.86(1.04-3.32)$ & $2.03(1.05-3.94)$ \\
\hline Missing & $2.35(1.24-4.44)$ & $2.12(1.07-4.18)$ & $7.83(2.79-21.95)$ & $8.89(2.88-27.43)$ \\
\hline Above low-income cut-off & Ref. & & & \\
\hline Stay in hospital during pregnancy but before labour & $1.49(1.01-2.19)$ & $1.39(0.95-2.05)$ & $1.54(0.76-3.11)$ & $1.17(0.57-2.41)$ \\
\hline \multicolumn{5}{|l|}{ Delivery type } \\
\hline Vaginal v. cesarean delivery & $0.91(0.66-1.27)$ & $0.91(0.64-1.29)$ & $0.79(0.39-1.60)$ & $0.82(0.38-1.76)$ \\
\hline Other birth complications versus no other birth complications & $1.14(0.68-1.89)$ & $1.16(0.69-1.96)$ & $1.42(0.42-4.79)$ & $1.36(0.38-4.85)$ \\
\hline $\begin{array}{l}\text { Infant admitted to NICU immediately postpartum versus no } \\
\text { immediate NICU admission }\end{array}$ & $1.43(0.93-2.19)$ & $1.37(0.89-2.11)$ & $2.78(1.21-6.40)$ & $2.92(1.25-6.80)$ \\
\hline
\end{tabular}

\$29999, have lower levels of education, be living at or below the before-tax low-income cut-off, have experienced abuse in the past year and to have been admitted to hospital prenatally compared with non-Indigenous Canadian-born mothers. We found that stressful events were more likely for Indigenous than non-Indigenous Canadian-born mothers only in the combined strata. In the rural strata only, Indigenous mothers were more likely to have experienced stressful events during pregnancy and less likely to be neutral about their travel experience compared with non-Indigenous Canadianborn mothers. No significant differences were noted between Indigenous and non-Indigenous mothers across geographic strata with respect to parity, birth satisfaction, delivery type, other birth complications or NICU admission.

In the total cohort (including mothers from small urban and rural areas but excluding those from large urban areas), $9.8 \%$ of Indigenous mothers travelled $200 \mathrm{~km}$ or more to give birth, whereas $2.0 \%$ of non-Indigenous mothers travelled $200 \mathrm{~km}$ or more to give birth (odds ratio [OR] 5.46, 95\% confidence interval [Cl] 3.52-8.48). Similarly, in rural areas, $23.2 \%$ of Indigenous mothers travelled $200 \mathrm{~km}$ or more to give birth, whereas $2.1 \%$ of
non-Indigenous mothers in rural areas travelled $200 \mathrm{~km}$ or more to give birth (OR 16.59, 95\% Cl 8.98-30.65). In small urban areas, the rates of travelling $200 \mathrm{~km}$ or more to give birth were similar between Indigenous (2.8\%) and non-Indigenous (1.9\%) mothers (OR 1.46, 95\% Cl 0.50-4.28).

Table 2 and Table 3 show the unadjusted and final adjusted stratified multivariable regression models for the evaluation of the associations between Indigenous and non-Indigenous identity and travel for birth in rural and small urban areas, respectively. Again, small numbers precluded regression modelling for large urban areas. In the models for rural areas, Indigenous mothers had higher odds of travelling $200 \mathrm{~km}$ or more to give birth compared with nonIndigenous Canadian-born mothers (adjusted OR 16.44, 95\% Cl 8.07-33.50). We found that admission to the NICU immediately after birth (adjusted OR 2.92, 95\% Cl 1.21-6.80) and before-tax income at or below the low-income cut-off (adjusted OR 2.03, 95\% Cl 1.053.94) were the only other variables independently associated with needing to travel $200 \mathrm{~km}$ or more for birth in the rural model.

In models for small urban areas, we found that Indigenous identity was not significantly associated with travelling $200 \mathrm{~km}$ or more 
Table 3: Unadjusted and adjusted multivariable odds of travelling $\mathbf{5 0 ~ k m}$ or more for birth among Indigenous and non-Indigenous Canadian-born mothers in small urban residency

Small urban area residence

$n=18300$

\begin{tabular}{|c|c|c|c|c|}
\hline \multirow[b]{2}{*}{ Variable } & \multicolumn{2}{|c|}{ Travel 50-199 km v. no travel or $<50$ km } & \multicolumn{2}{|c|}{ Travel $\geq 200 \mathrm{~km}$ v. no travel or $<50 \mathrm{~km}$} \\
\hline & $\begin{array}{l}\text { Unadjusted* OR } \\
\quad(95 \% \mathrm{CI})\end{array}$ & $\begin{array}{l}\text { Adjusted OR } \\
(95 \% \mathrm{Cl})\end{array}$ & $\begin{array}{l}\text { Unadjusted* OR } \\
\quad(95 \% \mathrm{CI})\end{array}$ & $\begin{array}{l}\text { Adjusted OR } \\
(95 \% \mathrm{Cl})\end{array}$ \\
\hline \multicolumn{5}{|l|}{ Ethnic identity } \\
\hline Indigenous v. non-Indigenous & $1.10(0.50-2.38)$ & $0.93(0.41-2.10)$ & $1.46(0.50-4.28)$ & $1.04(0.37-2.91)$ \\
\hline \multicolumn{5}{|l|}{ Age, yr } \\
\hline $15-24$ v. $25-50$ & $0.81(0.54-1.23)$ & $0.93(0.57-1.52)$ & $0.49(0.23-1.06)$ & $1.51(0.71-3.21)$ \\
\hline \multicolumn{5}{|l|}{ Marital status } \\
\hline Lone v. cohabitating & $1.21(0.69-2.13)$ & $1.26(0.64-2.50)$ & $1.01(0.35-2.91)$ & $0.82(0.26-2.56)$ \\
\hline \multicolumn{5}{|l|}{ Education } \\
\hline Less than high school & $1.54(0.83-2.86)$ & $1.70(0.81-3.57)$ & $2.17(0.61-7.74)$ & $2.02(0.44-9.33)$ \\
\hline High school diploma & $1.42(1.01-2.14)$ & $1.56(1.03-2.37)$ & $2.54(1.20-5.38)$ & $2.36(1.11-5.01)$ \\
\hline Post-secondary diploma and university & Ref. & & & \\
\hline \multicolumn{5}{|l|}{ Before-tax income } \\
\hline At or below low-income cut-off & $0.97(0.59-1.59)$ & $0.76(0.42-1.36)$ & $1.30(0.52-3.24)$ & $0.95(0.30-2.98)$ \\
\hline Missing & $0.51(0.14-1.90)$ & $0.40(0.10-1.55)$ & $0.01(0.01-0.01)$ & $0.01(0.01-0.02)$ \\
\hline Above low-income cut-off & Ref. & & & \\
\hline Stay in hospital during pregnancy but before labour & $2.52(1.67-3.79)$ & $2.20(1.42-3.40)$ & $3.26(1.55-6.83)$ & $2.56(1.24-5.31)$ \\
\hline \multicolumn{5}{|l|}{ Delivery type } \\
\hline Vaginal v. cesarean delivery & $0.82(0.57-1.20)$ & $0.81(0.55-1.20)$ & $0.67(0.30-1.46)$ & $0.72(0.30-1.75)$ \\
\hline $\begin{array}{l}\text { Other birth complications versus no other birth } \\
\text { complications }\end{array}$ & $1.96(1.16-3.30)$ & $2.02(1.14-3.59)$ & $0.99(0.26-3.71)$ & $1.05(0.26-4.31)$ \\
\hline $\begin{array}{l}\text { Infant admitted to NICU immediately postpartum } \\
\text { versus no immediate NICU admission }\end{array}$ & $1.80(1.11-2.91)$ & $1.53(0.92-2.55)$ & $1.96(0.73-5.27)$ & $1.74(0.58-5.18)$ \\
\hline
\end{tabular}

for birth (adjusted OR 1.04, 95\% Cl 0.37-2.91). Other covariates independently associated with travelling $200 \mathrm{~km}$ or more for birth among mothers in small urban areas were different from those in the analysis for rural areas, including high school versus postsecondary education level (adjusted OR 2.54, 95\% Cl 1.20-5.38) and prenatal hospital stay (adjusted OR 2.56, 95\% Cl 1.24-5.31) (Table 3).

In rural areas, we determined that the odds of travelling 50-199 km for birth were also higher for Indigenous mothers in unadjusted analysis (OR 1.89, 95\% Cl 1.01-3.53), but the association was no longer statistically significant in fully adjusted analysis (adjusted OR 1.63, 95\% Cl 0.83-3.22). Indigenous identity was not associated with travelling 50-199 km for birth for mothers living in small urban areas (adjusted OR 0.93, 95\% 0.41-2.10).

\section{Interpretation}

Our study provides additional evidence to quantify disparities in geographic access to birthing care for Indigenous compared with non-Indigenous Canadian-born people. Odds of Indigenous people travelling $200 \mathrm{~km}$ or more for birth were many times higher than those for non-Indigenous people. Our stratified geographic analysis showed that this disparity is specific to rural and remote regions. It is generally well understood in Canada that Indigenous people are more likely to live in rural and remote parts of the country than non-Indigenous people. For example, more than $70 \%$ of Inuit mothers live remotely in Inuit Nunangat. ${ }^{27}$ This knowledge, combined with well-documented and crosscutting disparities in Indigenous and non-Indigenous birth outcomes in Canada (including perinatal and neonatal infant death, ${ }^{28-30}$ preterm delivery, and both small-for-gestational-age and large-for-gestational-age infant birthweights) contributes to an assumption that the explanation for higher rates of travel for birth among Indigenous compared with non-Indigenous people is the resultant intersection of these factors. Access to maternity facilities and services in rural areas is commonly conceptualized as a joint problem of both Indigenous and non-Indigenous rural residents. ${ }^{3,5,7}$ Despite substantial qualitative and policy-oriented scholarship highlighting the need for Indigenous birth closer to 
home, there is a paucity of quantitative literature that evaluated Indigenous and non-Indigenous disparities in geographic access within rural areas.

Our findings show an Indigenous and non-Indigenous disparity in geographic access to birthing within rural areas and suggest that this disparity is not primarily driven by medical complications of pregnancy, birth complications requiring cesarean delivery or other birth complications. The need for NICU admission was significantly associated with travel for birth but did not significantly affect the disproportionate odds of long-distance travel for birth for Indigenous compared with non-Indigenous people who lived in rural areas. The most likely explanation for these disparities is persistent and systematic geographic inequities in the distribution of birthing facilities and providers across rural and remote regions of Canada, such that non-Indigenous rural residents and communities have more proximal geographic access compared with Indigenous residents and communities. ${ }^{31}$ These inequities have well-documented roots in colonial policies that favoured non-Indigenous settlements over First Nations, Inuit and Métis communities with respect to location of and access to quality health care facilities..$^{32,33}$ When facilities did exist for First Nations, Inuit and Métis, they were commonly segregated and second class. ${ }^{32,33}$

Given the size of the Indigenous and non-Indigenous disparity in access to birth close to home identified by our study, the documented negative impacts of birth away from home for Indigenous families, ${ }^{5-10}$ and existing recommendations, ${ }^{13-15}$ there is a clear need to advance policies that support more equitable geographic access to birthing for Indigenous families in rural areas. Future research that more precisely documents Indigenous and non-Indigenous inequities in the geographic distribution of birth facilities and services across rural areas at provincial, territorial, regional and small-area levels using geocoding will be required to inform and advance this work. Demonstrated strategies that could help improve access to birth close to home for Indigenous people include Indigenous midwifery, ${ }^{4,6}$ local education of Indigenous midwives and other Indigenous health professionals, ${ }^{34}$ and Indigenous leadership and participation in health service planning and delivery. ${ }^{35}$

\section{Limitations}

Although this study used the national MES data set, improving generalizability of findings across the country, there are several limitations. These include the exclusion of First Nations mothers living on reserve (representing $25 \%$ of the total Indigenous population in 2006) by the MES; the relatively small sample of First Nations, Inuit and Métis in the MES, which precluded provincial and territorial analyses owing to limitations of power; the use of the Canadian Census as the sampling frame, which may have contributed to biased and undersampling of Indigenous participants in urban areas; ${ }^{36}$ and the weighting of the Indigenous sample, which may not fully represent the geographic distributions of Indigenous populations across the country because of these other limitations. Finally, the MES data set is now 13 years old. Although our observed Indigenous and non-Indigenous disparities in travel for birth can be assumed to persist, since there has been no substantial expansion of Indigenous birthing facilities in or proximal to Indigenous communities during this time, there is a pressing need for follow-up Indigenous maternity experiences or broader reproductive health surveys that include First Nations people on reserve, incarcerated people and those whose children have been apprehended by child protection agencies.

\section{Conclusion}

Indigenous people in Canada experience inequities in access to birth close to home compared with non-Indigenous people. These inequities are localized to rural and remote areas of Canada, and undermine Indigenous family and community relationships at a critical time. To address these inequities and better align with international law (United Nations Declaration on the Rights of Indigenous Peoples, www.un.org/esa/socdev/unpfii/documents/ DRIPS_en.pdf), the Calls to Action of the Truth and Reconciliation Commission of Canada (www.trc.ca/assets/pdf/Honouring_the Truth_Reconciling_for_the_Future_July_23_2015.pdf) and the Calls for Justice of the National Inquiry into Missing and Murdered Indigenous Women and Girls (www.mmiwg-ffada.ca/final-report/), there is a pressing need to advance policies, facilities and services to increase geographic access to birth closer to home.

\section{References}

1. Owolabi T. Managing obstetrical patients during severe acute respiratory syndrome outbreak. J Obstet Gynaecol Can 2004;26:35-41.

2. Robinson M. Giving birth alone and away from home. The Star [Toronto] 2017 Jan. 1. Available: www.thestar.com/news/insight/2017/01/01/giving-birth -alone-and-far-from-home.html (accessed 2019 Dec. 17).

3. Hutcheon JA, Riddell CA, Strumpf EC, et al. Safety of labour and delivery following closures of obstetric services in small community hospitals. CMAJ 2017;189:E431-6.

4. Van Wagner V, Osepchook C, Harney E, et al. Remote midwifery in Nunavik, Québec, Canada: outcomes of perinatal care for the Inuulitsivik Health Centre, 2000-2007. Birth 2012;39:230-7.

5. Benoit C. Modern maternity care in Canada. Ottawa: Vanier Institute of the Family; 2015. Available: https://vanierinstitute.ca/modern-maternity-care-in -canada/ (accessed 2021 Apr. 19).

6. Simonet F, Wilkins R, Labranche E, et al. Luo. Primary birthing attendants and birth outcomes in remote Inuit communities - a natural "experiment" in Nunavik, Canada. J Epidemiol Community Health 2009;63:546-51.

7. Kornelsen J, McCartney K. The safety of rural maternity services without local access to cesarean section: an applied policy research unit review. Vancouver: University of British Columbia, Applied Policy Research Unit at the Centre for Rural Health Research; 2015. Available: www.perinatalservicesbc.ca/Documents/Resources/ SystemPlanning/Rural/SafetyRuralMaternityServicesWithoutLocalAccessCsection 2015.pdf (accessed 2019 Dec. 16).

8. Sharma S, Kolahdooz F, Launier K, et al. Canadian Indigenous women's perspectives of maternal health and health care services: a systematic review. Divers Equal Health Care 2016;13:334-48.

9. O’Driscoll T, Kelly L, Payne L, et al. Delivering away from home: the perinatal experiences of First Nations women in northwestern Ontario. Can J Rural Med 2011;16:126-30.

10. Kornelsen J, Kotaska A, Waterfall P, et al. The geography of belonging: the experience of birthing at home for First Nations women. Health Place 2010;16:638-45.

11. Lawford KM, Giles A, Bourgeault I. Canada's evacuation policy for pregnant First Nations women: resignation, resilience, and resistance. Women Birth 2018;31:479-88.

12. Position statement on evacuation for birth. Ottawa: National Aboriginal Council of Midwives; 2019. Available: https://indigenousmidwifery.ca/wp-content/ uploads/2019/05/PS_BirthEvac.pdf (accessed 2020 Oct. 8).

13. The First Nations health transformation agenda. Ottawa: Assembly of First Nations; 2017:1-137. Available: www.afn.ca/uploads/files/fnhta_final.pdf (accessed 2019 Dec. 16).

14. Kanatami IT. Social determinants of Inuit health in Canada. Ottawa: Inuit Tapiriit Kanatami; 2014:1-46. Available: www.itk.ca/wp-content/uploads/2016/07/ITK _Social_Determinants_Report.pdf (accessed 2019 Dec. 16). 
15. SOGC policy statement. No. 251, December 2010. Returning birth to Aboriginal, rural, and remote communities. J Obstet Gynaecol Can 2010;32:1186-8.

16. Ottawa to pay for travel companion for Indigenous women giving birth away from reserve. CBC News 2017 Apr. 9. Available: www.cbc.ca/news/politics/indigenous -women-pregnancy-reserve-escort-policy-change-1.4063082 (accessed 2019 Dec. 17).

17. Birthing services suspended in Rankin Inlet, says government. CBC News 2020 Aug. 4. Available: www.cbc.ca/news/canada/north/birthing-services-suspended -rankin-inlet-nunavut-1.5673683 (accessed 2020 Dec. 21).

18. Dzakpasu S, Kaczorowski J, Chalmers B, et al. the maternity experiences study group of the Canadian prerinatal surveillance system, Public Health Agency of Canada. The Canadian maternity experiences survey: design and methods. J Obstet Gynaecol Can 2008;30:207-16.

19. Chalmers B, Dzakpasu S, Heaman M, et al.; the Maternity Experiences Study Group of the Canadian Perinatal Surveillance System, Public Health Agency of Canada. The Canadian maternity experiences survey: an overview of findings. J Obstet Gynaecol Can 2008;30:217-28.

20. Kingston D, Heaman M, Chalmers B, et al. Comparison of maternity experiences of Canadian-born and recent and non-recent immigrant women: findings from the Canadian Maternity Experiences Survey. J Obstet Gynaecol Can 2011;33:1105-15.

21. Khanlou N, Haque N, Skinner A, et al. Scoping review on maternal health among immigrant and refugee women in Canada: prenatal, intrapartum, and postnatal care. J Pregnancy 2017;2017:8783294. [Epub ahead of print 2017 Jan. 22]. doi: 10.1155/2017/8783294.

22. Adams M, Alexander G, Kirby R, et al. Perinatal epidemiology for public health practice. New York: Springer; 2009.

23. Jolles D, Stapleton S, Wright J, et al. Rural resilience: the role of birth centers in the United States. Birth 2020;47:430-7.

24. Focus on Geography series. 2016 census. Ottawa: Statistics Canada; 2017. Cat no 98-404-X2016001. Available: www12.statcan.gc.ca/census-recensement/2016/as-sa/ fogs-spg/Facts-CAN-eng.cfm?Lang=Eng\&GK=CAN\&GC=01\&TOPIC=9 (accessed 2019 Dec. 19).

25. Population and dwelling count highlight tables. Ottawa: Statistics Canada; 2016; modified 2019 July 2. Available: www150.statcan.gc.ca/n1/daily-quotidien /171025/mc-a001-eng.htm (accessed 2021 Apr. 26).
26. Smylie J, Firestone M. Back to the basics: identifying and addressing underlying challenges in achieving high quality and relevant health statistics for Indigenous populations in Canada. Stat J IAOS 2015;31:67-87.

27. Map 1. Inuit population by residence inside or outside Inuit, Nunangat, 2016. Ottawa: Statistics Canada; 2016. Available: https://www12.statcan.gc.ca/census -recensement/2016/dp-pd/hlt-fst/pd-pl/Table.cfm?Lang=Eng\&T=703\&S=87\&O=A (accessed 2019 Dec. 19).

28. Sheppard AJ, Shapiro GD, Bushnik T, et al. Birth outcomes among First Nations, Inuit and Métis populations. Health Rep 2017;28:11-6.

29. Smylie J, Crengle S, Freemantle J, et al. Indigenous birth outcomes in Australia, Canada, New Zealand and the United States - an overview. J Womens Health (Larchmt) 2010;4:7-17.

30. Smylie J, Fell D, Ohlsoon A; Joint Working Group on First Nations, Indian, Inuit and Métis Infant Mortality of the Canadian Perinatal Surveillance System. A review of Aboriginal infant mortality rates in Canada - striking and persistent Aboriginal/non-Aboriginal inequities. Can J Public Health 2010;101: 143-8.

31. Access to health services as a social determinant of First Nations, Inuit and Métis Health. Prince George (BC): National Collaborating Centre for Aboriginal Health (NCCAH); 2011.

32. Geddes G. Medicine unbundled, a journey through the minefields of Indigenous health care. Vancouver: Heritage House; 2007.

33. Lux M. Separate beds: a history of Indian hospitals in Canada, 1920s-1980s. Toronto: University of Toronto Press; 2016.

34. Epoo B, Stonier J, Van Wager V, et al. Learning midwifery in Nunavik: communitybased education for Inuit midwives. Pimatziwin 2012;10:283-99. Available: https:// journalindigenouswellbeing.com/wp-content/uploads/2013/02/03EpooStonier.pdf (accessed 2021 Apr. 26).

35. Smylie J, Kirst M, McShane K, et al. Understanding the role of Indigenous community participation in Indigenous prenatal and infant-toddler health promotion programs in Canada: a realist review. Soc Sci Med 2016;150:128-43.

36. Rotondi MA, O'Campo P, O'Brien K, et al. Our health counts Toronto: using respondent-driven sampling to unmask census undercounts of an urban Indigenous population in Toronto, Canada. BMJ Open 2017;7:e018936.
Competing interests: Cheryllee Bourgeois is a midwife with Seventh Generations Midwives Toronto. Evelyn Harney George is a past Co-Chair and currently a Community Engagement Lead for the National Aboriginal Council of Midwives. No other competing interests were declared.

This article has been peer reviewed.

Affiliations: Well Living House (Smylie), St. Michael's Hospital; Dalla Lana School of Public Health (Smylie), University of Toronto; Ontario HIV Treatment Network (O'Brien); Midwifery Education Program (Bourgeois), Ryerson University; Seventh Generations Midwives Toronto (Bourgeois), Toronto, Ont.; Department of Public Health (Daoud), Faculty of Health Sciences, Ben Gurion University of the Negev, Beer-Sheva, Israel; National Aboriginal Council of Midwives (Harney George, Bebee), BC; Native Women's Association of Canada (Ryan), Ont.; Bridlewood Medical Centre (Beaudoin), Kanata, Ont.

Contributors: Janet Smylie led the study design, oversaw the data analyses and led the writing of all versions of the manuscript. Kristen O'Brien led the data analyses and writing of methods and results sections. Emily Beaudoin supported finalization of the literature review and tables. Nihaya Daoud contributed to the study design, data analyses and iterative versions of the manuscript. Cheryllee Bourgeois, Evelyn Harney George, Kerry Bebee and Chaneesa Ryan contributed to the interpretation of the study results and final versions of the manuscript. All of the authors gave final approval of the version to be published and agreed to be accountable for all aspects of the work.

Content licence: This is an Open Access article distributed in accordance with the terms of the Creative Commons Attribution (CC BY-NC-ND 4.0) licence, which permits use, distribution and reproduction in any medium, provided that the original publication is properly cited, the use is noncommercial (i.e., research or educational use), and no modifications or adaptations are made. See: https://creativecommons.org/licenses/by-nc-nd/4.0/

Funding: Janet Smylie was supported by a Canadian Institutes of Health Research Applied Public Health Research Chair while the manuscript was being produced and is currently a Tier 1 Canada Research Chair in Advancing Generative Health Services for Indigenous Populations in Canada.

Data sharing: The Canadian Maternity Experiences Survey data set is held by Statistics Canada and can be accessed by application to Canadian Research Data Centres. For more information on access see https://crdcn. org/research.

Accepted: Mar. 25, 2021

Correspondence to: Janet Smylie, janet.smylie@utoronto.ca 